\title{
The Effectiveness of Polarized Polychromatic Non-Coherent (BIOPTRON) Light in the Management of Acute Patellar Tendinopathy: A Case Report
}

\author{
Stasinopoulos Dimitrios* \\ Assistant Professor, Physiotherapy, Dept. of Physiotherapy, Faculty of Health and Caring Sciences, University \\ of West Attica, Greece
}

\begin{abstract}
*Corresponding Author: Stasinopoulos Dimitrios, Physiotherapy Bachelor, Assistant Professor, Physiotherapy, Dept. of Physiotherapy, Faculty of Health and Caring Sciences, University of West Attica, Greece.
\end{abstract}

\begin{abstract}
Background: The effectiveness of the physiotherapy modalities have been investigated in chronic patellar tendinopathy (PT). To our knowledge, there have been no studies to find out the effectiveness of physiotherapy modalities in chronic PT with acute pain. The aim of the present report is to find out the effect of polarized polychromatic non-coherent (BIOPTRON) light in chronic PT with acute pain. Case report: A patient with chronic right dominant unilateral PT with pain for about 24 hours participated in the present case study. The patient followed a course of BIOPTRON light twice per day for five consecutive days. Outcome measures were pain and function, using a VISA-P questionnaire. The evaluation of the patient occurred before and after the end of treatment. There was a decline in pain and a rise in function in all evaluations. Conclusion: The results of the present trial suggest that a course of Bioptron light as described in the present trial can produce significant improvements in terms of pain and disability in chronic PT with acute pain.
\end{abstract}

Keywords: patellar tendinopathy, Bioptron light, jumber's knee, patellar tendonitis, polarized polychromatic non-coherent light

\section{INTRODUCTION}

Patellar Tendinopathy (PT) commonly referred to as Jumper's knee or patellar tendonitis is the most common tendinopathy in the knee area. Pain and decreased function are the main symptoms of PT. Diagnosis is simple. The symptoms are reproduced by (1) lower limb activities such as squat or hop; (2) palpation on the site of pain (mainly at the inferior pole of the patella); and (3) clinical tests such as decline test [1].

The management of PT is usually conservative. A plethora of physiotherapy techniques have been recommended for the management of PT. The effectiveness of the recommended physiotherapy modalities has been investigated in chronic PT. Chronic PT is degenerative or failed healing tendon response rather than inflammatory [2]. To our knowledge, there have been no studies to find out the effectiveness of physiotherapy modalities in acute PT. More recently, physiotherapists are able to use a new modality called Polarized Polychromatic NonCoherent (BIOPTRON) Light. However, the evidence of the Bioptron Light in the management of PT, acute or chronic, is minimal. Therefore, the objective of the present report is to find out the effect of Bioptron Light in acute PT.

\section{Case Presentation}

The subject was a 24-year-old male basketball player with a six- month history of anterior knee pain, in his right knee (dominant limb). He was diagnosed as having chronic PT. He has played basketball for about ten years. The site of pain was over the inferior pole of the patella without spreading down. However, he complained of acute pain (less than 24 hours) in the anterior aspect of his rightkneein the last visit. The acute pain was only on the inferior pole of the patella. $\mathrm{He}$ had intensive training about $24 \mathrm{hrs}$ ago. He experienced acute pain immediately after 
finishing training. Since then, he complained of constant pain. He was not able to sleep. He did not receive any NSAIDs. He did not have any other symptoms or any problems in peripheral joints or in the spine. In theisotonic resisted knee extension the power was 1 on the Oxford scale and there was pain over the inferior pole of the patella, $9 / 10$ on the VAS. The reported pain of the patient in the decline test was $9 / 10$ on the VAS. All the other movements (passive, active and under resistance movements of the low back, hip and ankle) were pain free, with full range of motion and strength. All ligamentous stress tests were normal, meniscal stress tests were normal, muscle strength tests were normal and no capsular pattern was found. Knee extension by gliding the patella medially was negative, without reproducing the pain; furthermore the position of the patella was normal [3]. These two latter procedures ruled out the patellofemoral joint dysfunction. Finally, the acute pain was reproduced by palpation over inferior pole of the patella.

Patient received Bioptron light therapy, via the BIOPTRON Pro optical device (BIOPTRON AG, Wollerau, Switzerland;) [4] for 10min twice a day (morning and afternoon) for five consecutive days providing ten sessions in total [5]. The equipment used was noninvasive. The physical parameters of the light output from this optical medical device were as follows: wavelength $480-3400 \mathrm{~nm}$, light spot size $254 \mathrm{~cm}^{2}$, and specific power density $40 \mathrm{~mW} / \mathrm{cm}^{2}$. During active light exposure, the energy output of the device (energy density) was $2.4 \mathrm{~J} / \mathrm{cm}^{2}$ $/ \mathrm{min}$ (that is, $24 \mathrm{~J} / \mathrm{cm}^{2} / 10 \mathrm{~min}$ session). During each light exposure the patient was seated and fitted with darkened eye wear, the treatment area (knee area) was exposed, carefully cleaned (by wiping with a sterile gauze soaked in clean water) and dried, then the optical medical device was powered up and used to 'paint' the exposed area with Bioptron light for $10 \mathrm{~min}$. During light exposure, the optical medical device was positioned via a support floor stand to be approximately at right angles to the skin surface, and at a distance of approximately $10 \mathrm{~cm}$ from the treatment area. A "beep" signified the end of the $10 \mathrm{~min}$ treatment.

Pain and function were measured in the present study. The patient was evaluated at the baseline and at the end of treatment. The VISA-P questionnaire was used to monitor the pain and function of patient. The instrument is a simple questionnaire, consisted of eight questions that takes less than five minutes to complete and once patients are familiar with it they will be able to complete most of it themselves. It is a valid and reliable outcome measure for patients with patellar tendinopathy [6].

\section{RESUltS}

VISA-P score was 19 at the initial evaluation. At the end of the treatment (session 10), there was a rise in VISA - P score of 57 units (Table $1)$.

Table1. VISA -P score before and after evaluation

\begin{tabular}{|c|c|}
\hline & VISA - P \\
\hline Beforetreatment & 19 \\
\hline Aftertreatment & 76 \\
\hline
\end{tabular}

\section{DISCUSSION}

The present case study has looked at the effect of Bioptron light in a patient experiencing chronic PT with acute pain and its findings have demonstrated significant improvements in terms of pain and disability. The results obtained from this case report are novel; as to date, similar studies have not been conducted.

The most common physiotherapy treatment for PT is a supervised or in clinic exercise program [7]. However, the chronic PT patient with acute pain was not able to perform an exercise program due to pain. The patient was advised by the clinician to follow an exercise program after completing the Bioptron light treatment.

Like Low Level Laser Light (LLLL), Bioptron light is also a low-power light source, but differ from LLLL in that Bioptron light is incoherent and polychromatic rather than coherent and monochromatic [4]. Moreover, Bioptron light combines infrared light at a wavelength of 700$3400 \mathrm{~nm}$ and visible light at a wavelength of 480-700 nm [4]. In contrast, LLLL contains either infrared or visible light at one specific wavelength [4]. Several drawbacks have impaired the usefulness of LLLL in comparison to Bioptron light, such as high risk, required user skills, the small diameter of the laser beam, which allows only a limited area to be treated and high cost [4].

Both the infrared and visible parts of the electromagnetic spectrum of Bioptron light can explain its mechanism of action [4]. It is probable that Bioptron light has biostimulative effects improves the blood supply and accelerates the cellular mechanisms [4], but more research is needed to investigate how this occurs. 
Since pain relief and improvements in function were noted in the present case study on a short term, it is proposed that Bioptron light may potentially have promoted an anti-inflammatory effect in the soft tissues [4]. However, to understand the potential changes to the tissues in response to Bioptron light therapy, future studies should consider employing outcome assessments that are capable of monitoring the changes in deeper tissues.

It is important to mention that no side effects were reported during or after the treatment period. There is no UV light in the BIOPTRON light spectrum, so there is no tanning or heating effect on the skin.[4] Furthermore, BIOPTRON light is not harmful to the eyes, and poses no danger to pregnant women.[4] It is easy-to -use $\&$ can be used in the clinical setting or if required in the patient's home.[4] Finally, BIOPTRON light is not associated with cancer: the unsafe range for cancer risk is UV light at $250 \mathrm{~nm}$, and the shortest wavelength in the Bioptron spectrum is 480nm.[4]

The present case study was the first report to examine the effectiveness of Bioptron light on chronic PT with acute pain. Previous research assessed the effectiveness of Bioptron light on chronic conditions such as Lateral Elbow Tendinopathy (LET) $[8,9]$ and carpal tunnel syndrome $[10,11]$. However, acute and chronic conditions are two different conditions and the results are not comparable. In addition, one controlled study assessed the effectiveness of Bioptron light in acute ankle sprains [12], but ankle sprain is a ligament injury whereas LET is a tendon injury. Therefore, ligament injury and tendon injury are two different conditions and the results are not comparable. Finally, one case report examined the effectiveness of Bioptron Light on acute LET [5]. The previously reported trials found that a course of Bioptron light may improve patients' symptoms. The findings of these trials encourage the design of future welldesigned RCTs that might produce strong evidence for the effectiveness of Bioptron light on sports / musculoskeletal injuries.

A course of Bioptron light treatment was applied in the present study based on manufacturers' claims [4]. It is a dose-response modality and the optimal treatment dose has obviously not yet been discovered. Future studies are needed to standardize Bioptron light parameters in the management of PT (acute, chronic and calcific) and on other sports / musculoskeletal conditions.

Although the positive effects of such a treatment approach in chronic PT with acute pain have been reported in the present study, its study design limits the generalization of these results. Future well-designed trials are needed to confirm the results of this case report establishing the effectiveness of such a treatment approach in the management of PT(acute, chronic and calcific). In addition, structural changes in the tendons related to the treatment interventions are needed to investigate. Further research is needed to establish the cost-effectiveness of such treatment, because reduced cost is an important issue for the recommendation of any given treatment and the possible mechanism of action of this treatment approach [4].

\section{CONClusion}

A course of Bioptron light treatment had reduced the pain and improved the function in a chronic PT patient with acute pain at the end of the treatment. Further well-designed trials to confirm the results of the present case study are needed.

\section{REFERENCES}

[1] Kountouris A, Cook J. Rehabilitation of Achilles and patellar tendinopathies. Best Pract Res Clin Rheumatol. 2007; 21: 295-316.

[2] Stasinopoulos D. Patellar Tendinopathy May not Be the Proper Term for Patients With Clinical Diagnosis of Patellar Tendon Disorder. Trauma Mon. 2014; 19: e15301.

[3] McConnell J. The management of Chondromalacia Patellae. A long-term solution. Australian Journal of Physiotherapy. 1986; 32: 24-32.

[4] Ioannis M, Michalis T, Dimitrios L, et al. Tendinopathy: The Role of Polarised Polychromatic Non-Coherent Light Commonly called Bioptron Light. J Prev Inf Cntrl. 2016, 2:2

[5] Stasinopoulos D. The Effectiveness of Polarized Polychromatic Non-Coherent (BIOPTRON) Light in the Management of Acute Lateral Elbow Tendinopathy: A case report Biomed J Sci \& Tech Res14 (3) 2019. BJSTR. MS.ID.002562

[6] Visentini PJ, Khan KM, Cook JL, Kiss ZS, Harcourt PR, Wark JD. The Visa score: An index of severity of symptoms in patients with jumper's knee (patellar tendinosis). Victorian institute of sport tendon study group. J Sci Med Sport. 1998; 1: 22-28. 
[7] Malliaras P, Cook J, Purdam C, Rio E. Patellar Tendinopathy: Clinical Diagnosis, Load Management, and Advice for Challenging Case Presentations. J Orthop Sports Phys Ther. 2015; 45: 887-898.

[8] Stasinopoulos D, Stasinopoulos I Comparison of effects of Cyriax physiotherapy, a supervised exercise programme and polarized polychromatic non-coherent light (Bioptron light) or the treatment of lateral epicondylitis. Clin Rehabil 2006; 20:12-23

[9] Stasinopoulos D, Stasinopoulos I, Pantelis M, Stasinopoulou K Comparing the effects of exercise program and low-level laser therapy with exercise program and polarized polychromatic non-coherent light (bioptron light) on the treatment of lateral elbow tendinopathy. Photomed Laser Surg 2009; 27:513-20
[10] Stasinopoulos D, Stasinopoulos I, Johnson MI Treatment of carpal tunnel syndrome with polarized polychromatic noncoherent light (Bioptron light): A preliminary, prospective, open clinical trial. Photomed Laser Surg 2005; 23:225-8

[11] Dimitrios S, Stasinopoulos L. Treatment of Carpal Tunnel Syndrome in pregnancy with Polarized Polychromatic Non-coherent Light (Bioptron Light): A Preliminary, Prospective, Open Clinical Trial. Laser Therapy 2017; 26(4), 289-295

[12] Stasinopoulos D, Papadopoulos C, Lamnisos D, Stasinopoulos I. The use of Bioptron light (polarized, polychromatic, non-coherent) therapy for the treatment of acute ankle sprains. DisabilRehabil 2017; 39:450-457.

Citation: Stasinopoulos Dimitrios. The Effectiveness of Polarized Polychromatic Non-Coherent (BIOPTRON) Light in the Management of Acute Patellar Tendinopathy: A Case Report. ARC Journal of Clinical Case Reports. 2020; 6(2):10-13. doi:dx.doi.org/ 10.20431/2455-9806.0602002.

Copyright: (C) 2020 Authors. This is an open-access article distributed under the terms of the Creative Commons Attribution License, which permits unrestricted use, distribution, and reproduction in any medium, provided the original author and source are credited. 